\title{
Der russische Vielvölkerstaat zwischen Zentralismus und Föderation
}

\author{
Z.R. DITTRICH
}

Wenn man den Werdegang des russischen Staates seit dem späten Mittelalter bis heute aus staatswissenschaftlicher oder verfassungsrechtlicher Sicht betrachtet, kann man nicht umhin, der Revolution von 1917 die Bedeutung eines radikalen Umbruchs beizumessen. Das alte Zarenreich war während seiner jahrhundertelangen Existenz ein zentralistischer Einheitsstaat, dessen inneres Machtsgefüge es geradezu erforderte, seine damals in der Mehrheit (57\%) fremdstämmige Bevölkerung behördlicherseits zu einer uniformen Untertanenmasse umzuschmelzen und womöglich zu russifizieren. Die aus der Oktoberrevolution hervorgegangene Staatsbildung in der Gestalt der Union der sozialistischen Sowjetrepubliken (1922) dagegen kennzeichnet sich durch eine bundesstaatliche Struktur, die, vom Grundsatz der Selbstbestimmung der Völker ausgehend, sowohl die einzelnen Gliedstaaten als auch die autonomen Nationalrepubliken, Gebiete (Oblast) und Bezirke (Okrug) nach dem Kriterium ihrer Ethnizität ausgestaltet hat. In der jüngsten Sowjetverfassung vom Oktober 1977 wird ausdrücklich anerkannt, dass die fünfzehn Teilstaaten souveräne Republiken sind (Art. 76) und dass sie das Recht auf Sezession besitzen (Art. 72). ${ }^{1}$ Im Vergleich also mit der vorrevolutionären Lage kann man sich kaum einen grosseren Unterschied vorstellen, und die amtlichen Kreise in der Sowjetunion weisen denn auch gern und nachdrücklich auf die neue Struktur hin. Der sozialistische Föderalismus, wie er in der USSR verwirklicht wurde, gilt noch immer als musterhafte Lösung für die Probleme eines Vielvölkerstaates, zumindest aus der Sicht der kommunistischen Propaganda.

Der historischen Forschung, die sich an erster Stelle für das tatsächliche Funktionieren der Heerschaftssysteme der Vergangenheit und der Gegenwart interessiert, fallt es freilich nicht schwer, aus der Gegenüberstellung der staatsrechtlichen Theorie und der politischen Praxis, wie diese durch die Quellen und alltag-

1. Konstitucija (osnovnoj zakon) sojuza sovetskich socialisticeskich respublik, Englische Übersetzung: Constitution (Fundementat Law) of the Union of Soviet Socialist Republics (Moskow, 1977) 59 und 57; H. Carrère d'Encausse, 'Determinants and Parameters of Soviet Nationality Policy', in J.R. Azrael, Soviet Nationality Policies and Practices (New York-London, 1978) 39 ff. 


\section{Z.R. DITTRICH}

liche Beobachtungen ermittelt ist, Schlüsse zu ziehen, welche die vorgeblich riesengrosse Kluft zwischen dem ehemaligen Zarenreich und der heutigen Sowjetföderation weitgehend als optische Täuschung erweisen. Bezüglich des alten Russland hat schon Anfang der fünfziger Jahre Georg von Rauch in seiner ungemein anregenden Arbeit: Russland, staatliche Einheit und nationale Vielfalt.

Föderalistische Krafte und Ideen in der russischen Geschichte meiner Meinung nach überzeugend dargelegt, dass die russische Monarchie nur bedingt einen Einheitsstaat darstellte, und dass es im Laufe ihrer Geschichte schon immer Kräfte, Tendenzen und Faktoren gegeben hat, die der offiziellen Zentralisierung nicht ohne Erfolg entgegenwirkten. Fraglich ist jedoch, ob man sie deswegen schon als föderalistisch bezeichnen kann, in dem Masse etwa, wie von Rauch es getan hat. Um diese Problematik zu klären, ist es notwendig, die Wesensmerkmale des autokratischen Herrschaftssystems, das in Russland bis 1905 ungeschmälert fortbestanden hatte, näher in Augenschein zu nehmen. Die uneingeschränkte, unteilbare und unmittelbare Gewalt, von den russischen Selbstherrschern seit Iwan dem Schrecklichen beansprucht, stellte einen höheren Grad persönlicher Machtenfaltung dar als ihn die absoluten Monarchien westlicher Herkunft je erreicht hatten. ${ }^{3}$ Es ist jedenfalls zweckdienlicher, die russische Autokratie als eine Abart der orientalischen Despotie zu betrachten. ${ }^{4}$ Der Zar vereinigte ja nicht bloss die höchste weltliche und geistliche Autorität in sich; was die von den Ideen des byzantinischen Mönchs Agapetos geprägte Moskauer Staatslehre dem Zaren an Verfügungsgewalt über seine Untertanen zuerkannte, kam der Macht Gottes gleich - der Zar sei irdischer Gott (zemnyj Bog)! Obwohl die russische Bevölkerung in viele Ränge und soziale Schichten gegliedert war, die untereinander grosse Unterschiede in Rechtslage und Wohlstand aufwiesen, war dem Zaren gegenüber jedermann ein Sklave, dessen Stellung gänzlich von der Gnade des Herrschers abhing und daher unsicher blieb. Erst die Gnadenurkunde Katharinas II. (1785) gab dem russischen Adel einigen Rechtsschutz auch gegen monarchische Willkür und leitete - reichlich spät - die Entwicklung zur ständischen Gesellschaftsordnung ein. Ein auf wechselseitiger Treue und Loyalität beruhendes Verhältnis zwischen dem Herrscher und dem Untertan, wie es die westlichen Monarchien kannten, war der russischen Autokratie fremd. Diese Herrschaftsform ging von der strikt patriarchalen Autoritätsauffassung aus, dass der Zar und von ihm ermächtigten Gewaltträger den Untertanen gegenüber keine

2. (Veröffentlichungen des Osteuropa-Institutes München, Band 5; München, 1953).

3. Grundlegend H. Neubauer, Car und Selbstherrscher, Beiträge zur Geschichte der Autokratie in Russland (Wiesbaden, 1964) 20 ff.; B. Nørretranders, The Shaping of Czardom under Ivan Groznyj (Copenhagen, 1964) $87 \mathrm{ff}$.

4. Z.R. Dittrich, 'Wittfogel and Russia. On the Origin of Russian Autocracy', Acta Historiae Neerlandica, I (Leiden, 1966) $53 \mathrm{ff}$. 
Pflichten haben konnten, die Untertanen ihrerseits aber der Obrigkeit einen bedingungslosen Gehorsam schuldeten. Dementsprechend musste die Autorität vorzugsweise Furcht einflössen; der psychische Druck, dem die Bevölkerung ständig ausgesetzt war, variierte von der kirchlichen Vorstellung der strafenden Allmacht bis zur massiven Einschüchterung durch willkürliche Gewaltakte, die ganze Gegenden und soziale Schichten trafen.

Unter diesen Umständen war die einzige, freilich hochwirksame Beschränkung der Selbstherrschaft und des Selbstherrschers die kulturell, organisatorisch und technisch bedingte Unzulänglichkeit des Machtsapparates, der zudem im riesigen, jedoch sehr dünn bevölkerten Zarenreiche enorme Entfernungen zu bewältigen hatte. Bekanntlich rechtfertigte Katharina II. in ihrer 'Grossen Instruktion' die autokratische Regierung in Russland mit zwei Argumenten: 1. bei der Grossräumigkeit des Reiches gewährleiste nur sie eine rasche Erledigung der Staatsgeschäfte; und 2, es sei jedenfalls besser, einem einzigen Herrn als vielen zu gehorchen. ${ }^{5}$ In beiderlei Hinsicht war die Kaiserin jedoch Opfer eigener Wunschbilder. Die Effizienz der Selbstherrschaft und ihrer Organe war in Wirklichkeit recht mangelhaft, da die rückständige Agrargesellschaft einfach nicht imstande war, ein hinreichend starkes, geschultes und bezahltes Berufsbeamtentum hervorzubringen. Die sprichwörtlichen Übel der russischen Bürokratie, ihre Ignoranz, ihre Bestechlichkeit und ihre Gewohnheit des Machtsmissbrauchs zwangen die Herrscher, immer neue Kontrollinstanzen und Aufsichtsverfahren einzuführen, die dann doch nur vorübergehend ihrem Zweck gerecht werden konnten. ${ }^{6}$ Auch um die Homogenität der autokratischen Regierung war es nicht so gut bestellt, wie Katharina II. sich träumen mochte. Es lag ja im eigenen Interesse des Selbstherrschers, zwischen seinen Mitarbeitern, den einzelnen Ämtern und sogar ganzen Zweigen öffentlicher Dienste den Geist des Misstrauens und der Rivalität zu fördern, um gefährliche Machtskonzentrationen zu verhüten, so dass er selbst die tatsächliche Entscheidungsgewalt behielt. Damit hängt auch zusammen, dass die Autokratie die Führung wichtiger Dienststellen lieber Kollegien als einzelnen Personen anvertraute. Andererseits war nicht zu verhindern, dass sich innerhalb der Beamtenschaft, besonders in der Provinz, auf die Dauer verschiedene Interessentengruppen bildeten, deren einziges Anliegen es war, die eigene Machtposition zäh zu verteidigen. Der Selbsterhalfungstrieb solcher Gruppen bewirkte, dass jede Neuerung und insbesondere jede Verwaltungsreform auf Widerstand und Sabotage bis zur völligen Verhinderung rechnen musste. Kurz und gut: Die autokratische regierung war bei aller äusserlichen Straffheit und trotz einkalkuliertem

5. Polnoe Sobranie Zakonov, XVIII, No. 12949, Glava II, 9-12, $122 \mathrm{ff}$.

6. M. Raeff, 'The Russian Autocracy and its Officials', Harvard Slavic Studies, IV (Den Haag, 1957) 77 ff; S. Frederick Starr, 'Tsarist Government: the Imperial Dimension', in Azrael, Soviet Nationality, $3 \mathrm{ff}$. 


\section{Z.R. DITTRICH}

und recht kostspieligem Machtsaufwand und echter Grausamkeit in Wirklichkeit ein schwerfälliger und schlecht funktionierender Apparat, der wegen seiner inneren Gegensätze immer wieder mit allerlei ad-hoc-Massnahmen, Kunstgriffen und peinlichen Umbildungen repariert werden musste, um den Erfordernissen einer zeitgemässen Verwaltung wenigstens einigermassen zu genügen.

Vor diesem Hintergrund bekommt die Frage nach etwaigen Möglichkeiten des Föderalismus im alten Russland zwangsläufig einen zwiespältigen Charakter. Einerseits ist evident, dass die Selbstherrschaft ihrer Natur nach keine Gewaltenteilung, keinen wirklichen Pluralismus innerhalb ihres Machtsbereiches dulden konnte. Notwendigerweise neigte sie zum Unitarismus und Zentralismus, und in mancher Hinsicht versuchte sie diese Tendenzen bis zum Äussersten durchzusetzen. Andererseits war der autokratische Staatsapparat offensichtlich nicht fähig, während der säkularen Ausdehnung des Reiches, das schon seit dem sechzehnten Jahrhundert fremde Völker einbezog, die neuerworbenen Gebiete mit dem alten Kern restlos zu verschmelzen. So entstand die Verwaltungsform, die man meines Erachtens nach noch am besten als einen unvollkommenen, etappenmässigen Zentralismus bezeichnen kann. Das alte Reichsgebiet, politisch und sozial gleichgeschaltet und einheitlich verwaltet, wurde von einem breiten Gürtel unterschiedlich organisierter Gebiete umgeben; da gab es Provinzen mit besonderem Verwaltungsstatut bis zu Schutzstaaten unter der Regierung eigener Dynastien. Das Verhältnis zwischen dem Kern und den Randgebieten war, wie schon angedeutet, dynamischer Art. Gegenüber den unaufhörlichen Bestrebungen, den von der Zentrale aus vollkontrollierten Bereich immer weiter auszudehnen und die Randgebiete stärker in den Griff zu bekommen, standen partikularistische Kräfte und Hemmnisse nicht zuletzt auch verwaltungstechnischer Natur, welche den auf die Dauer wohl unaufhaltsamen Aufmarsch des Einheitsstaates in Einzelsektoren immerhin wirksam bremsen konnten.

Die geschichtliche Entwicklung Russlands bietet manches Beispiel für die eigenartigen Beziehungen zwischen dem Kernland und den Randgebieten. Die Teilung des Reiches in Opricnina und Zemšcina unter Iwan dem Schrecklichen war offensichtlich ein Versuch, diesem Nebeneinander eine dualistische Form zu geben. ${ }^{7}$ Der alte Moskauer Kern wurde 1565 in ein dominium speciale umgewandelt, wo der Zar die bestehenden sozialen Zustände und Besitzverhältnisse rücksichtslos und mit massiver Gewaltanwendung ganz nach eigenem Ermessen umgestaltete, wobei die Aristokratie als führende Schicht vernichtet und durch den Dienstadel ersetzt wurde. Die Zemšcina, in der die bisherigen Zustände nicht wesentlich geändert wurden, umfasste dagegen die westlichen und östlichen Randgebiete, und es war wahrscheinlich kein Zufall, dass Iwan für diesen Teil den Tatarischen

7. M. Hellmann, Iwan der Schreckliche (Göttingen, 1968) 59 ff. 
Vasallenfürst christlichen Glaubens Simeon Bekbulatovic zu seinem Nebenzaren ernannte. Nachdem Iwan in seinen letzten Regierungsjahren das Experiment der Zweiteilung aufgegeben hatte, wurde es nie widerholt. Die Unterschiede zwischen dem Moskauer Kern und den Randgebieten blieben an sich fortbestehen. Indessen dehnte sich der Bereich der vollzentralisierten Verwaltung durch die Bauernkolonisation besonders an der Wolga und in Südrussland ständig aus, die einstigen Randgebiete wurden also assimiliert und zugleich wurden als Folge der kriegerrischen Entwicklungen in Europa und der ungläublich raschen Durchdringung Sibiriens neue Landschaften angelagert, welche die Funktion des Randes übernahmen.

Unter den Romanows wurde die Lage allerdings durch den Umstand kompliziert, dass territoriale Neuerwerbungen am westlichen Saum des russischen Reiches nicht ohne vertragliche Zusicherungen an die betroffène Bevölkerung stattfanden. So bestätigte der später heissumstrittene Vertrag von Perejaslavl (1654) dem ukrainischen Kosakentum seine Freiheiten und so erhielten die Ostseeprovinzen Estland und Livland von Peter dem Grossen (1710) die vollständige Anerkennung ihrer ständischen Selbstverwaltung. Alexander I. verfuhr bei der Einverleibung Finnlands (1809) und Bessarabiens (1812) und bei der Schöpfung des Kongressköningreichs Polen (1815) auf ähnliche Weise. Obwohl Alexander I. der einzige russische Herrscher war, der sich ernsthaft bemühte, sein Imperium pluralistisch zu gestalten, zeigte sich bereits während seiner Regierung, dass auch eine völkerrechtlich abgesicherte Garantie den Bewohnern der Randprovinzen keinen wirksamen Schutz gegen die Übergriffe der zentralen Behörden bieten konnte. Die Autokratie und das von ihr geistig geprägte Beamtentum betrachteten die besonderen Rechte der autonomen Gebiete noch immer als jederzeit selbstherrlich widerrufbare Gnadenakte. Und auch da, wo solche Rechte formell gültig blieben, führte die tagliche Praxis der St. Petersburger Amtskreise zu ihrer tatsächlichen Aushöhlung. Hinzu kam im Laufe des neunzehnten Jahrhunderts die allmähliche Entfaltung des grossrussischen Nationalismus, der den ohnehin schon kräftigen Zentralisierungsbestrebungen neue Impulse gab. Immer mehr wurde der Abbau der Sonderstellung der ursprünglich autonomen Gebiete zu einer planmässigen Russifizierung, die jedoch bei der betroffenen Bevölkerung eine oft leidenschaftliche Gegenreaktion auslöste. An die Stelle des regionalen Partikularismus der alten Oberschicht trat der Nationalismus der unterjochten Völker, die im zaristischen Russland ein riesiges Völkergefängnis erblickten und nur durch ganzliche Loslösung ihrer Heimatlander die Nationalfrage lösen zu können glaubten.

Den Föderalismus, der als ideologisch begründetes Streben nach Verwirklichung bundesstaatlicher Verfassung verstanden wird, gab es in Russland kaum vor dem Ende des achtzehnten Jahrhunderts. Unter dem Eindruck des amerika- 
nischen Unabhängigkeitskrieges schwärmte A.N. Radiscev zwar von einer Föderation, wobei ihm das tragische Schicksal Alt-Nowgorods zur scharfen Verurteilung des Moskauer Unitarismus inspirierte. Von den verschiedenen Verfassungsentwürfen, die von Mitarbeitern Alexanders I. verabschiedet wurden, lässt sich generell sagen, dass sie bloss eine Neuordnung der Staatsverwaltung bezweckten, wobei den einzelnen Gouvernements eine gewisse, meist sehr bescheidene Autonomie zugestanden werden sollte. Selbst das Verfassungsprojekt des Senators Novosil'cov (1819), das Russland in zehn Generalstatthalterschaften einteilte, kann eher als eine Dezentralisierung gewertet werden; ${ }^{8}$ aber was soll man von diesem Reformvorschlag halten, wenn dessen Urheber im Kongresskönigreich als Exponent russischen Beamtentums die polnische Selbstverwaltung sabotierte! Freilich: der Gedanke einer bundesstaatlichen Verfassung nach dem Beispiel der Vereinigten Staaten hat unter den Dekabristen Anklang gefunden, besonders bei dem gemässigten nördlichen Zweig ihrer Bewegung. Im Verfassungsentwurf N.M. Muravjovs, der dreizehn autonome Gliedstaaten (derzavy) und zwei Regionen (oblasti) vorsah, sollte die bundesstaatliche Reform hauptsächlich als Mittel wirken, um die Machtvollkommenheit der Selbstherrschaft zu durchbrechen. Die geplanten Gliedstaaten waren zum Teil künstliche geographische Neubildungen; eine Zergliederung des Reiches nach ethnischem Prinzip lag Muravjov offensichtlich fern. ${ }^{9}$ In der südrussischen geheimen Genossenschaft der Vereinigten Slawen, die den Dekabristen nahe stand, träumte man dagegen von einer allslawischen Föderation, deren Gliedstaaten zwar auf nationaler Grundlage beruhten, in der aber dem grossrussischen Volke, das nun einmal das weitaus stärkste war, die führende Rolle zufiele. Schon im Anfangsstadium des russischen Föderalismus also sah man sich der kaum lösbaren Aufgabe gegenüber, aus einem sehr grossen und mehreren kleinen Partnern einen lebensfähigen und dennoch wirklich pluralistischen Bundesstaat zu bilden. Dass im Kreise der Verschwörer übrigens auch ganz andere Auffassungen lebten, zeigt die Russkaja Pravda des Führers des radikalen Südbundes P.I. Pestel', der die künftige russische Republik als einen nationalrussischen Einheitsstaat betrachtete, in dem Fremdstämmige rücksichtslos russifiziert werden sollten. ${ }^{10}$.

Nach dem Scheitern des Staatsstreichs vom Dezember 1825 wie auch des ersten polnischen Aufstandes 1830/31 folgte im Zarenreich die Ära einer harten Reaktion. Erörterung etwaiger Staatsreformen, zumal im föderalistischen Sinne, war auf amtlicher Ebene schlechterdings unmöglich geworden; nur die in den Untergrund gedrängte Opposition konnte weiterhin den Gedanken des Föderalismus

\footnotetext{
8. M. Raeff, Plans for PoliticalReform in Imperial Russia 1730-1905 (Englewood Cliffs, 1966) 110

ff.

9. M. Raeff, The Decembrist Movement (Englewood Cliffs, 1966) $110 \mathrm{ff}$.

10. Ibidem, $124 \mathrm{ff}$.
} 
pflegen. Von den Auffassungen der Vereinigten Slawen ausgehend, hat der Verfasser des politischen Programms der Kyrill- und Methodiusgesellschaft zu Kiew (1846-47), der Historiker N.J. Kostomarov (1817-85), die Vereinigung aller Slawen in einem Bund freier Nationalrepubliken gefordert, wobei auch die Ukrainer endlich Autonomie erlangen sollten. ${ }^{11}$ Der Autor, der als nationalbewusster Ukrainer ein feines Gespür für die Gefahr eines übermächtigen Grossrussentums entwickelt hatte, wollte in der künftigen Föderation das Übergewicht dieses Volkes möglichst verringern. So sollte Kiew die Hauptstadt sein und die russische Sprache sollte nur eine lingua franca, nicht aber die Amtssprache des Slawenbundes werden. Das grossrussische Volk sollte ferner nicht einen einzigen Gliedstaat bilden, sondern in vierzehn geographisch bedingte Teilgebiete eingeteilt werden und sozusagen einen Bundesstaat innerhalb des Bundesstaates darstellen. Diese Konstruktion, die einigermassen den Zustand nach 1922 vorwegzunehmen scheint, verfolgte unmissverständlich den Zweck, den Kräfteverhältnissen im allslawischen Bunde eine gewisse Ausgewogenheit zu verleihen.

Ausser Kostomarov, der sich in seinem späteren Leben übrigens ausdrücklich von seinem bundesstaatlichen Programm lossagte, haben auch andere Regimekritiker aus der Nikolaitischen Ära, wie Herzen, Bakunin und PetraSevsky, jeder auf seine Weise föderalistische Ideen befürwortet. Begreiflicherweise hatte diese Problematik im Kreise der damaligen russischen Opposition jedoch bei weitem nicht die Priorität, welche der Bauern- oder Verfassungsfrage zuerkannt wurde. Das zeigte sich deutlich gerade während der reformfreundlichen Regierung Alexanders II. (1855-81). Während sich die Öffentlichkeit lebhaft mit einer Vielzahl aktueller Themen auseinandersetzte, überliess sie die Diskussion über die Frage Bundesstaat - Einheitsstaat den Historikern, die sie im Rahmen einer Würdigung der altrussischen Vergangenheit führten. Die 1864 eingeführte Zemstwo-Selbstverwaltung, die übrigens nur 37 von den 51 Gouvernements erfasste, lief in zweierlei Hinsicht den bundesstaatlichen Bestrebungen zuwider. Einerseits kanalisierte sie das Interesse der Öffentlichkeit in Richtung auf einen harmlosen Provinzialismus, der eher ein Gehilfe denn ein Nebenbuhler der Zentralbehörden und ihres Verwaltungsapparates war, andererseits machte sie die gefährlichen Konsequenzen sichtbar, die jede ernsthafte Dezentralisierung im damaligen Russland haben musste, nämlich den durch die agrarischen Sonderinteressen bedingten Partikularismus der Grundbesitzer, der neuen Tendenzen in der Wirtschaft und Gesellschaft sicher mehr im Wege stand als die St. Petersburger Bürokratie. Auch der Umstand, dass der zweite polnische Aufstand (1863) in der russischen Gesellschaft eine Woge leidenschaftlichen Nationalismus auslöste, war der Ver-

11. S. Frederick Starr, Decentralisation and Self-Govemment in Russia 1830/70 (Princeton, 1972) $100 \mathrm{ff}$. 
breitung föderalistischen Gedankengutes ohnehin ungünstig. Wenn man sich überhaupt in der Opposition mit föderalistischen Lösungen wie im Besonderen mit der heiklen polnischen Frage befasste, handelte es sich meistens um Sonder-1 ösungen, die die Struktur des russischen Staates eigentlich nicht berührten und deswegen für die Föderalismus-Thematik ohne Bedeutung waren.

Die einzige nennenswerte Ausnahme in dieser Periode bildet ein KostomarovSchüler, M.P. Dragomanov (1841-95), ein Ukrainer wie sein Lehrer. ${ }^{12}$ Man darf anmerken, dass dies wohl kein Zufall war. In den damaligen Verhältnissen waren die Ukrainer, deren nationale Differenzierung vom Grossrussentum ein schwieriger und langwieriger Vorgang war, noch lange nicht so weit, dass sie ein völlig unabhängiges Dasein hätten erstreben können. Eine föderalistische Umgestaltung Russlands schien immerhin eine machbare Lösung ihres ukrainischen Problems zu sein. Wegen seiner fortschrittlichen Gesinnung in die Emigration gedrängt, entfaltete Dragomanov von Genf aus eine rege journalistische Tätigkeit in russischer und ukrainischer Sprache. In mehreren Arbeiten, denen ein umfassendes Studium schweizerischer und nordamerikanischer Verhältnisse vorangegangen war, widmete er seine Aufmerksamkeit der bundesstaatlichen Neugestaltung Russlands. Um der Gefahr einer Majorisierung durch das Grossrussentum vorzubeugen, sah Dragomanov bewusst von der Bildung einzelner Gliedstaaten auf ethnographischer Grundlage ab. Das ganze Reich ohne Finnland sollte in zwanzig Regionen eingeteilt werden, deren Umfang und Form hauptsächlich von geographischen und wirtschaftlichen Bedingungen bestimmt sein und die gemeinsam eine lockere Föderation nach schweizerischem Modell bilden sollten. Innerhalb der Gliedstaaten-Kantone wollte Dragomanov eine Vielzahl von kleineren Distrikten (Volost') auf ethnographischer Grundlage entstehen lassen, denen er eine weitgehende Selbstverwaltung, hauptsächlich im kulturellen Bereich, gewähren wollte. Der künftige Bundesstaat nach Dragomanovs Auffassung sollte ferner eine bikamerale Volksvertretung haben: Neben der Abgeordnetenkammer, von der Gesamtbevölkerung gewählt, sollte eine Kammer der Vertreter der Nationalitäten stehen. Charakteristisch für dieses zweistufige Föderalismusmodell war der den grösseren Völkern Russlands auferlegte Verzicht auf Bildung eigener, die ganze Nation umfassender Verwaltungseinheiten, wodurch einerseits das natürliche Übergewicht des Grossrussentums zumindest kompensiert wurde, andererseits jedoch nationale Einheitsbestrebungen der Ukrainer, Polen, Balten und so weiter auf das Erlangen einer kulturellen Autonomie eingeschränkt wurden. Die Partei der Sozialisten-Revolutionäre, die 1902 in ihrem Programm die Konzeption Dragomanovs übernahm, radikalisierte sie in dem Sinne, dass sie den Randvölkern Russlands, die sich auf Grund der Selbstbestimmung von dem Ge-

12. S.V. Utechin, Ceschichte der politischen Ideen in Russland (Stuttgart, 1966) $144 \mathrm{ff.}$ 
samtstaat lösen wollten, das Recht auf völlige Abtrennung ausdrücklich zubilligte und die bundesstaatliche Struktur auf das Restgebiet beschränkte.

Die politische Lage des Zarenreiches am Ende des neunzehnten Jahrhunderts kennzeichnete sich durch eine zunehmende Verschärfung der nationalen und gesellschaftlichen Gegensätze. Die allgemein repressive Grundhaltung der verkrampften Selbstherrschaft führte in nationaler Hinsicht zu einer rücksichtslosen Russifizierung, welche die nichtrussischen Völker Europas und Asiens mit einer Steigerung ihrer nationalen Bestrebungen im Sinne des Nationalismus, der Irredenta und separatistischer Bewegungen aller Art beantworteten. Gleichzeitig wurden durch die beginnende Industrialisierung die sozialen Verhältnisse komplizierter, indem der chronischen Notlage des Bauerntums und der wachsenden Ungeduld der Intelligenz auch noch die explosive Kraft des jungen städtischen Proletariats hinzugefügt wurde. Für die russische Arbeiterbewegung, die von Anfang an von sozialistischen und im engeren Sinne marxistischen Auffassungen geprägt wurde, war das Gemisch nationaler und sozialer Gegensätze eine Elementartatsache, der sie weder auf dem Gebiet der Theorie noch in der politischen Praxis ausweichen konnte. Dabei boten die Grundsätze, Auffassungen und Organisations- und Kampfformen, wie sie sich inzwischen unter der Arbeiterschaft westeuropäischer Länder gebildet hatten, einen nur ungenügenden Anhalt, da sie zu sehr auf die dort herrschenden Verhältnisse zugeschnitten waren, und die grundlegende historisch-materialistische Ideologie, wie sie von Marx, Engels und ihren Jüngern ausgearbeitet wurde, manche Unklarheit und selbst Widersprüchlichkeit in sich barg. Während die einen, die sich übrigens auf die Grundsatzerklärung der Zweiten Internationale von 1896 berufen konnten, eine restlose Verwirklichung der nationalen Selbstbestimmung forderten, wodurch sie sich eindeutig mit dem Nationalismus der unterdrückten Völker solidarisierten, konnten die anderen in Namen des proletarischen Internationalismus jede Äusserung nationaler Tendenzen verurteilen und ein allmähliches Aufgehen der einzelnen Völker in eine uniforme Menschheit mit einer Universalsprache für gesetzmässig und wünschenswert halten. Zwischen diesen beiden Äussersten gab es noch manche Varianten, wie namentlich die austromarxistische Lösung, welche die bundesstaatliche Verfassung mit kultureller Autonomie verknüpfte. Hinsichtlich der polnischen und jüdischen Frage mit ihren spezifischen Aspekten wurden die russischen Marxisten schon auf dem Gebiet der Partei- und Gewerkschaftsorganisation zur Stellungnahme gezwungen.

Es ist nicht unsere Aufgabe, die Auseinandersetzungen über die nationale Frage und die Standorte einzelner Gruppen und Persönlichkeiten innerhalb der russischen Sozialdemokratie zu behandeln. Hinsichtlich der späteren Entwicklung genügt es, die Position Lenins und seiner Fraktion-Partei der Bolschewiki zu erörtern. ${ }^{13}$ Bei seiner schon früh formulierten Auffassung der Partei als einer 


\section{Z.R. DITTRICH}

militanten Vorhut der Arbeitersklasse und als eines elitären harten Kerns mit militärischer Disziplin und konspirativer Arbeitsweise war es nur logisch, dass Lenin in allen die Partei berührenden Fragen als konsequenter Verfechter des Zentralismus auftrat und die Einheitlichkeit der Organisation besonders gegen die nationalen Gliederungs- und Spaltungstendenzen verteidigte. ${ }^{14}$ Sein Bekenntnis zum Grundsatz der nationalen Selbstbestimmung auf dem Zweiten Kongress der RSDAP (1903) und auch später noch ist daher als ein mehr oder weniger aus taktischen Gründen erfolgtes Lippenbekenntnis zu betrachten. Erst die türbulenten Ereignisse der ersten russischen Revolution von 1905, wo neben der Bauernschaft auch die unzufriedenen Nationalitäten eine betrachtliche Rolle als Unruhefaktor spielten, veranlassten Lenin, seine ursprünglich negative Haltung allmählich zu revidieren. Wie bei der Bauernfrage, bei der er von der einseitig auf die Industrie und das städtische Proletariat fixierten Haltung der Sozialdemokratie Abstand genommen hatte, sah er die national-revolutionäre Bewegung der fremdstämmigen Einwohner Russlands als einen wertvollen Bundesgenossen im Kampf gegen den Zarismus. ${ }^{15}$ Dementsprechend wurde 1912-13, mit publizistischer Schützenhilfe von J.V. Stalin, die bolschewistische Politik in der Nationalfrage neu formuliert. Die Förderer des Föderalismus in den sozialistischen Parteien, besonders diejenigen, welche die austromarxistische Lösung befürworteten, wurden jetzt links überholt, in dem Sinne, dass Lenin die nationale Selbstbestimmung fast ausschliesslich als Recht auf staatliche Trennung interpretierte und den Eindruck erweckte, als wolle er das ganze russiche Reich in eine Anzahl unabhängiger Nationalstaaten auflösen. Die verbissenen Angriffe auf Föderalisten jedert Art, wodurch die Radikalität der bolschewistischen Stellungnahme unterstrichen werden sollte, dürfen uns aber nicht über die Tatsache hinwegtauschen, dass Lenin in puncto Partei ein Zentralist geblieben war und dass das Recht auf Selbstbestimmung und Abtrennung, das er augenscheinlich jedem Volke zubilligte, in seiner Auffassung so verklausuliert war, dass es in der Wirklichkeit nur in Ausnahmefällen in Anspruch genommen werden konnte: dann nämlich, wenn jenes Recht den Interessen der Werktätigen nicht widersprach. Bei der äusserst flexiblen Anwendung der Dialektik, die es Lenin ermöglichte, in Bezug auf die nationale Frage manchmal recht überraschende Positionen zu verteidigen, ist es nicht verwunderlich, dass auch ein Teil seiner Jünger, Bucharin

13. S. Shaheen, The Communist (Bolshevik) Theory of National Self-Determination. Its Historical Evolution up to the October Revolution (Den Haag, Bandung, 1956) $20 \mathrm{ff}$.

14. M. Holdsworth, 'Lenin on the Nationalities Question', in L. Schapiro R. Reddaway, Lenin, a Reappraisal (New York, Washington, London, 1967) 265 ff; R.A. Lewis, R.H. Rowland, R.S. Clem, Nationality and Population Change in Russia and the USSR. An Evaluation of Census Data 18971970 (New York, Washington, London, 1976) $110 \mathrm{ff}$.

15. R. Pipes, The Formation of the Soviet Union (Cambridge Mass., 1954) $35 \mathrm{ff}$. 
und Pjatakow und wohl auch der Renommier-Georgier Stalin, seinen Gedankensprüngen offensichtlich nur mit Mühe folgen konnten.

Der Ausbruch des Ersten Weltkrieges hat im Zarenreiche den Nationalbestrebungen der nichtrussischen Völker einen neuen Impuls gegeben. Das Polenmanifest des Grossfürsten Nikolaj Nikolajevic leitete die Phase von Versprechungen, taktischen Winkelzügen und Zugestandnissen ein, wobei die russische Regierung in zunehmendem Masse der Tatsache Rechnung tragen musste, dass sowohl ihre Bundesgenossen als die feindlichen Zentralmächte den nationalen Bestrebungen der Polen, Finnen, Balten, Ruthenen und Weissrussen wohlwollend gegenüberstanden und deren Vertretern in der Emigration und in den besetzten Westgebieten Gelegenheit gaben, sich politisch zu aussern. ${ }^{16}$ Auch Lenin, der durch den Kriegsausbruch zur durchgreifenden Umformulierung seines Programms gezwungen wurde, widmete sich erneut der nationalen Frage. Ende 1916 nahm er sich vor, eine grössere Arbeit über diesen Gegenstand zu schreiben, wobei die Nationalitatenproblematik im Sinne seiner neuen Imperialismustheorie erläutert werden sollte. Infolge stürmischer Ereignisse in Russland sah er sich aber gezwungen, die Arbeit auf halbem Wege abzubrechen.

Inzwischen wurden durch die Märzrevolution von 1917, der eine allgemeine Freiheitseuphorie folgte, Kräfte entfesselt, die auch die rückständigsten Völkerschaften des russischen Reiches zur Artikulierung ihrer nationalen Wünsche anregten. Der Umstand, dass die Vorläufige Regierung von Anfang an die Macht mit den Räten (Sovjets) teilen musste, wodurch die Lage immer undurchsichtiger und verworrener wurde, bot den Fremdstämmigen eine nahezu ideale Möglichkeit, sich im lokalen und regionalen Bereich als politische Potenz durchzusetzen. Schritt für Schritt wurden der Vorläufigen Regierung, in welcher die bürgerlichen Demokraten die Nachhut des grossrussischen Nationalismus und der zentralistischen Einheitsstaatauffassung bildeten, beträchtliche Zugeständnisse abgerungen; erst von den Polen, dann von den Finnen und danach von den Ukrainern, was schon einen deutlichen Umschwung in die Richtung des Föderalismus bedeutete. Gleichzeitig setzte sich der Vorgang der Lockerung der Staatseinheit auf der unteren Ebene, auf der die regionalen und örtlichen Räte in den Vordergrund traten, immer schneller durch, wenn auch nahezu alle nichtrussischen Völker damals nicht mehr als eine Kulturautonomie oder politische Selbstverwaltung im Rahmen des russischen Reiches erstrebten. Die endgültige Lösung der nationalen Frage sollte nach allgemeiner Meinung der Konstituante vorbehalten bleiben. ${ }^{17}$

Wie schon gesagt, hoffte Lenin durch eine radikale Formulierung des Selbstbe-

16. W. Conze, Polnische Nation und deutsche Politik (Köln, Graz, 1958); W. Basler, Deutschlands Annexionspolitik in Polen und im Baltikum 1914-1918 (Berlin, 1962); P. Borowsky, Deutsche Ukrainepolitik 1928 (Lübeck, Hamburg, 1970) $33 \mathrm{ff}$.

17. Von Rauch, Russland, 197. 


\section{Z.R. DITTRICH}

stimmungsrechts der Völker bis zur vollständigen Loslösung und Bildung eigener Nationalstaaten die latente Unzufriedenheit der nichtrussischen Bevölkerung für seine revolutionären Ziele auszunützen. Nicht ohne Schwierigkeiten gelang es ihm im April 1917 und in der Folgezeit, seine Partei auf diese Taktik einzuschwören. Übrigens erwartete Lenin, wie aus seinen damaligen Äusserungen klar hervorgeht, dass der Fortgang der proletarischen Revolution in Russland und anderswo die Werktätigen der nichtrussischen Völker dazu bewegen würde, das Selbstbestimmungsrecht im Sinne einer freiwilligen Wiedervereinigung auszuüben. Die Selbstbestimmungsparole, von den Bolschewiki in der Zeitspanne von März bis November 1917 verkündet, war unter den nichtrussischen Völkern indessen kaum werbewirksam. Gerade dort, wo die nationale Frage im Vordergrund stand, hatten diejenigen Parteien, welche die Volksbestrebungen gewissermassen verkörperten - wie die Sozialisten-Revolutionäre der Ukraine, die Menschewiki Georgiens und verschiedene nationale beziehungsweise religiöse Parteien in asiatischen Regionen - einen deutlichen Vorsprung, den sie während der ganzen Periode und auch später den Bolschewiki gegenüber behielten. Dies wurde ganz deutlich nach der Novemberrevolution, welche die Entwicklung von der Kulturautonomie und dem Föderalismusgedanken bis zur vollständigen Loslösung der Randgebiete Russlands nur beschleunigte.

Die neue Sowjetregierung, in der der Georgier Stalin als Volkskommissar für Nationalitätenfragen zuständig wurde, sah sich vor die Aufgabe gestellt, die programmatischen Versprechungen der Bolschewiki auch bezüglich der Nationalitaten einzulösen. In der am 15. November 1917 erlassenen Deklaration an die Völker Russlands wurde allen diesen Völkern ohne Rücksicht auf Grosse und Kulturstufe die Gleichheit, Souveränität und Selbstbestimmung 'bis zur Loslösung und Bildung eigener freier Staaten' feierlich zugestanden, einschliesslich des Rechtes, sich gegebenenfalls mit Gewalt zu befreien. Wo dieses Recht von den nichtbolschewistischen Landesregierungen oder Machthabern tatsächlich in Anspruch genommen wurde, in Finnland, in der Ukraine, in Bessarabien, in Kaukasien und so weiter, zeigte sich jedoch bald, dass die Sowjetregierung nicht die Absicht hatte, den sogenannten bürgerlichen Nationalisten die staatliche Loslösung zu gewähren. ${ }^{18}$ Unter dem Vorwand, dass nur die Werktätigen zu entscheiden hätten, wurde versucht, mit Hilfe oft winzig kleiner bolschewistischer Kerne und mit ihnen verbundener linksradikaler Elemente in den von Loslösung bedrohten Gebieten gewaltsam eine revolutionäre Herrschaft, die tatsächlich nur die Ausdehnung des sowjetrussischen Machtsbereiches bedeutete, einzuführen. So kann man sagen, dass die Randgebiete des ehemaligen Zarenreiches, die sich nach der Novemberrevolution tatsächlich aus dem russischen Staatsverband los-

18. E.H. Carr, The Bolshevik Revolution 1917-23, I (London, 1966) $292 \mathrm{ff}$. 
gelöst haben, ihr Ziel nur nach einem blutigen Kampf gegen die Sowjetregierung und ihrer Helfershelfer erreicht haben. Die gewaltsame Rückeroberung Georgiens im Frühjahr 1921 zeigt am deutlichsten, wie weit Lenin c.s. in der dialektischen Verdrehung eigener Erklärungen, Grundsätze und Vertragspflichten gehen konnten, wenn die Gelegenheit gunstig schien, die Herrschaft der Bolschewiki auszudehnen.

Schon kurz nach der bolschewistischen Machtsübernahme wurde ziemlich geräuschlos der Beschluss gefasst, dem künftigen Arbeiter- und Bauernstaat eine bundesstaatliche Struktur zu geben, und zwar auf der Grundlage seiner ethnischnationalen Gliederung. Die neue Verfassung, im Frühling 1918 eingeführt, verwandelte Russland in die russische sozialistische föderative Sowjetrepublik (RSFSR), wobei der tatsächlichen Bürgerkriegslage entsprechend noch keine bestimmten Regelungen zur Einteilung und Abgrenzung der Gliedstaaten festgesetzt wurden. In der Prawda vom 3. April 1918 wies der Volkskommissar Stalin darauf hin, dass die russische Föderation sich wesentlich von der Schweiz und den Vereinigten Staaten unterscheide; sie sei ja eine Vereinigung historisch geprägter Gebietsteile, welche sich durch die Lebensweise und Sprache ihrer Einwohner voneinander unterschieden. ${ }^{19}$ Wichtig war auch, dass die Föderation seiner Auffassung nach eine Übergangsform darstellte: die Gliedstaaten, die damals erst noch konstituiert werden müssten, sollten in der Zukunft in eine sozialistische Union aufgehen. So lassen sich schon in der Anfangsphase der bolschewistischen Diktatur zwei charakteristische Merkmale des Sowjetföderalismus hervorheben. Durch den Leitsatz, die einzelnen Gliedstaaten seien auf Grund ethnischer Unterschiedlichkeit zu bilden, hat man sich in Wirklichkeit für einen Bundesstaat Bismarckschen Typs entschieden. Da das grossrussische Volk, dessen absolutes zahlenmässiges Gewicht durch den Abfall der westlichen Randgebiete relativ schwerer als bisher wog, nun etwa die Hälfte der Bevölkerung des Gesamtstaates bildete, musste es, in einem Gliedstaat vereint, die übrigen Völker überragen und gegebenenfalls auch beherrschen, so dass die Föderation von vornherein nur ein Scheingebilde war. Der Schein trügt umso mehr, wenn von der bereits erwähnten Auffassung ausgegangen wird, dass die einzelnen Teilgebiete im Laufe der Entwicklung - und zwar gut marxistisch: im Laufe einer unvermeidlichen, weil gesetzmässigen Entwicklung - in eine neue Einheit integriert werden müssten. Dem Aufblühen (rascvet) der einzelnen Völker folge ihre wechselseitige Annäherung (sblizenie) und schliesslich die Amalgamierung (slijanie).

Wäre es bei einer ausgewogeneren Aufgliederung des russischen Reiches, etwa im Geiste Kostomarovs und Dragomanovs, nicht möglich gewesen, der bundes-

19. J.V. Stalin, Socinenija, IV, $66 \mathrm{ff}$. 
staatlichen Verfassung der RSFSR und (seit 1922) der Sowjetunion mehr Substanz zu geben? Ich neige dazu, diese Möglichkeit zu verneinen. Man kann ja nicht an der Dualität vorbeigehen, die dem kommunistischen Machtsystem eigen ist und die sich schon während des Bürgerkrieges in Russland voll ausgebildet hat, nämlich der Dualitat von Staat und Partei. ${ }^{20}$ Die letztgenannte, Trägerin der ungeteilten reolutionären Gewalt, gemäss der Doktrin des demokratischen Zentralismus streng hiërarchisch gegliedert und zentralistisch gelenkt, hat den nachrevolutionären Staatsapparat, den sie auf den Trümmern der alten Ordnung allmählich aufbaute, durch zahlreiche persönliche und institutionelle Bande an sich gekettet und zum verlässlichen Instrument eigener Herrschaft gemacht. Folglich muss doch wohl eine echte Selbstverwaltung auf regionaler und örtlicher Ebene, geschweige denn in bundesstaatlichen Organen, schlechthin ausgeschlossen sein die zentralistische Partei manipuliert ja überall und immer Beamte, Funktionäre und Volksvertreter zugunsten einer einheitlichen politischen Stellungnahme. Also blieb der Föderalismus im Sowjetstaate von vornherein auf eine besondere gliedstaatliche Verwaltung beschränkt, die jedoch nicht etwa autonom wirkt, sondern im Gegenteil die Weisungen der Zentrale ausführt. Der Föderalismus tut sich im übrigen nur durch den Gebrauch der eigenen Sprache in Behörden, Betrieben und Schulen kund.

Die Bildung autonomer nationaler Republiken, Regionen (Oblast) und Bezirke (Okrug) innerhalb der RSFSR geschah schrittweise in den zwanziger Jahren und wurde nachträglich noch mehrmals von der zentralen Regierung revidiert; erst seit etwa zwei Jahrzehnten kann dieser Vorgang als abgeschlossen gelten. Die heutige RSFSR zahlt 16 autonome Republiken neben den 49 Provinzen (Kraj oder Oblast' genannt), in die das restliche Grundgebiet eingeteilt ist. Kleinere nationale Einheiten, wie die schon erwähnten autonomen Gebiete und Bezirke, sind dagegen verwaltungsmässig den Provinzen, beziehungsweise den Republiken, untergeordnet. Bei der Einrichtung nationaler Gebietseinheiten spielte die zahlenmässige Grösse oder die Kulturstufe der betroffenen Völker grundsätzlich keine Rolle. Entscheidend war, dass ein Volk ein mehr oder weniger zusammenhangendes Territorium bewohnte, wobei die oft winzig kleinen Nationalitäten des hohen Nordens, die über riesige Flachen verbreitet sind, wegen ihrer Lage einigermassen bevorzugt wurden. Die Abgrenzung folgte ungefähr den ethnographischen Trennlinien. In manchen Fällen wurde die Grenzziehung so perfektionistisch durchgeführt, dass bizarre Linien mit eingestreuten Enklaven und Exklaven zustandekamen. Solche Absurditäten wurden später bereinigt, beispielsweise im Süden der Baschkirischen ASR. In anderen Fällen wurden zwei oder gar mehrere Nationalitäten (Dagestan) in einer einzigen Verwaltungseinheit zusam-

20. W. Leonhard, Sowjetideologie heute, II, Die politischen Lehren (Frankfurt a/M, 1974) $169 \mathrm{ff}$. 
mengefügt, obwohl es durchaus möglich gewesen ware, ihnen gesonderte Nationalbezirke zu geben. Man findet in der RSFSR auch einige Republiken, Oblasti und Okrugi, wo die namengebende Nationalität nur eine Minderheit der Bevölkerung darstellt; das krasseste Beispiel sind wohl die Juden in Birobidschan mit einem Bevölkerungsanteil unter 10\%. Andererseits leben heute ungefähr $80 \%$ der Tataren ausserhalb der Tatarischen ASR und müssen die 1,900.000 Deutschen und 1,100.000 Polen, zahlenmässig starke und höchstentwickelte Nationen, wohl nicht nur wegen ihrer Streulage jegliche Form einer Gebietsautonomie entbehren.

Die RSFSR, deren Gliederung auf der Landkarte einigermassen an die Zustände im Wilhelminischen Reich erinnert, kann als ein Bundesstaat im Bundesstaate bezeichnet werden. In den Randgebieten des ehemaligen Zarenreiches, die sich nach der Novemberrevolution von Sowjetrussland getrennt und zeitweilig als unabhängige Nationalstaaten konstituiert hatten (die Ukraine, Weissrussland, Georgien, Aserbeidschan, Armenien und so weiter) wurde nämlich auch nach der Rückeroberung durch die Sowjets hauptsächlich aus politisch-opportunistischen Gründen die formale Eigenstaatlichkeit nicht abgeschafft. Statt diese Gebiete der RSFSR einfach als autonome Republiken einzuverleiben, wurde eine neue Form der Wiedervereinigung gewählt: die Union der sozialistischen Sowjetrepubliken, deren Gründung Ende 1922 zwischen der RSFSR und der Ukraine, Weissrussland und der damaligen transkaukasischen Föderation vereinbart wurde. Die Verfassung der neuen Union von 1923 stellte die auswärtigen Beziehungen, Landesverteidigung und Verkehrsangelegenheiten unter die ausschliessliche Kompetenz des Bundes, während zum Beispiel für die Justiz oder die Volksbildung ursprüngslich nur die Gliedstaaten zuständig bleiben sollten. Diese vertikale Kompetenzteilung zwischen der Union und den Republiken einschliesslich der RSFSR konnte jedoch unter den gegebenen politischen Machtverhältnissen aufdie Dauer nicht standhalten. Durch eine Reihe gesetzlicher wie administrativer Änderungen, die in der jüngsten Verfassung von 1977 ihren vorläufigen Abschluss gefunden haben, wurden die Regierungen der Union und der einzelnen Gliedstaaten weitgehend gleichgeschaltet und mit denselben Kompetenzbereichen ausgestattet, jedoch in dem Sinne, dass die Unionsorgane den gliedstaatlichen übergeordnet sind und deren Tätigkeit im grossen und ganzen bestimmen. So hat heute jeder der fünfzehn Gliedstaaten einen eigenen Aussenminister und besitzt jeder Gliedstaat kraft Art. 80 der Verfassung sogar das Recht, mit Drittländern diplomatische Beziehungen zu pflegen und Verträge zu schliessen ${ }^{21}$; es gibt jedoch keinen einzigen Bereich, in welchem ein Gliedstaat unabhängig von der Unionsregierung seine Angelegenheiten regeln könnte.

21. Constitution (Fundamental Law) 61. Für die Verhältnisse unter der Verfassung von 1936 G. Meyer, ed., Das politische und gesellschaftliche System der UdSSR. Ein Quellenband (Köln, 1976) 43 ff. Für die heutige Lage J.F. Hough, M. Fainsod, How the Soviet Union is governed (Cambridge Mass., London, 1979) $480 \mathrm{ff}$. 


\section{Z.R. DITTRICH}

Was die interne Verwaltung anbelangt, sind die meisten Sowjetrepubliken Einheitsstaaten. Nur Georgien, Aserbeidschan, Uzbekistan und Tadschikistan haben zusammen vier autonome Republiken und drei autonome Regionen innerhalb ihrer Grenzen. ${ }^{22}$ Deren Stellung ist die gleiche wie die der Republiken und Regionen der RSFSR, was in der neuen Verfassung auch dadurch hervorgehoben wird, dass sie in Art. 85 und 87 nebeneinander erwähnt werden. Man kann sich vorstellen, dass eine derart komplizierte bundesstaatliche Struktur, wie es sie in der USSR schon länger als ein halbes Jahrhundert gibt, unter anderen politischen Machtsverhältnissen kaum einen einzigen Tag funktionieren könnte. Nur weil die kommunistische Partei mit ihrer zentralistisch-hierarchischen Struktur und eisernen Disziplin den Staatsapparat in allen seinen Gattungen und Zweigen bis ins einzelne kontrolliert, sind die Widersprüche zwischen den verfassungsrechtlich souveränen Gliedstaaten und der zentralen Bundesgewalt und die Gegensätze der unklaren, sich oft überschneidenden Kompetenzen der einzelnen Regierungsinstanzen und Volksvertretungen bisher ohne Auswirkungen geblieben. Wenn man sich diese Lage vergegenwärtigt, wird unschwer die beinahe erstaunliche Kontinuität erkennbar, welche die scheinbar föderativ und praktisch als Einheitsstaat regierte USSR mit dem ehemaligen Zarenreiche verbindet. ${ }^{23}$

Ein wesentlicher Unterschied bleibt jedoch unabdingbar: anders als unter den Zaren gibt es heute nationale Verwaltungseinheiten, die den nichtrussischen Völkern der Sowjetunion zumindest in sprachlicher Hinsicht ihre Eigenart garantieren. Dieses Erbstück aus der Gründerzeit der Sowjetunion, als begeisterte Mitarbeiter des Narkomnaz auch die rückständigsten Völker Eurasiens mit eigener Schriftsprache samt Rechtschreibung und Alphabet beglückten und als den in der Diaspora lebenden Juden eine neue Heimat in Birobidschan zuteil wurde, konnte selbst in den schlimmsten Jahren der Schreckensherrschaft Stalins nicht zurückgenommen werden. Eine gesetzliche Diskriminierung der Fremdstämmigen gibt es seit 1917 nicht mehr und die offene und gewaltsame Russifizierung gehört der Vergangenheit an. Indessen ist die nationale Frage in der Sowjetunion nicht etwa zur allgemeinen Zufriedenheit gelöst, wie die offizielle Propaganda behauptet; im Gegenteil: wenn die Verhältnisse um das Jahr 1925 zum Massstab genommen werden, lässt sich in mancher Hinsicht eine Verschlechterung feststellen, zudem sind infolge der oft überstürzten gesellschaftlichen Entwicklung neue Probleme, neue Schwierigkeiten, neue Gefahren hervorgetreten. So wurden die historisch verwurzelten Unterschiede zwischen den einzelnen Völkern der USSR im Laufe der sechs Jahrzehnte seit der revolutionären Staatsgründung

22. Wobei allerdings die zu Aserbeidschan gehörige Nachitschewan ASR eine Exklave bildet.

23. Bemerkenswert S. Frederick Starr, 'Tsarist Government: the Imperial Dimension', in Azrael, Soviet Nationality, $6 \mathrm{ff}$. 
nicht etwa überwunden, sondern eher verfestigt und sogar verhärtet. Während sich das Grossrussentum in der Rolle der staatstragenden Nation im grossen und ganzen behauptet und schon dank seiner Sprache manche praktischen Vorteile geniesst, gibt es Völker, deren Stellung als unterprivilegiert gewertet werden kann: es wären die immer noch vertriebenen Krimtataren, ferner die zerstreut lebenden Polen und Deutschen zu nennen. ${ }^{24}$ Widerspruchsvoll bleibt die Lage der Sowjetjuden, die in intellektuellen Berufen noch immer mit einem unverhältnismässig hohen Anteil vertreten sind, im Alltagsleben aber manche Beschränkungen und Erniedrigungen zu erleiden haben ${ }^{25}$ Sind alle solche Mängel irgendwie noch der nachwirkenden Vergangenheit anzurechnen, als schwerwiegender betrachte ich die neuen Formen der Gefährdung der nationalen Eigenart nichtrussicher Völker. An erster Stelle gibt es da die offizielle Förderung eines gesamtsowjetischen Volksbewusstseins, wobei den Fremdstämmigen die russische Sprache als zweite Muttersprache aufgedrungen wird. Es ist selbstverständlich, dass der gesamtsowjetische Patriotismus grossrussische Züge aufweist und eine schleichende Russifizierung bewirkt, zumindest in dem Sinne, dass es das nationale Sondergefühl der Nichtrussen zurückdrangt und die Abwehr russischer Kulturpenetration unter die Sanktionen unzulässiger oder gar verräterischer Handlungen stellt. Vielleicht noch verhängnisvoller wirken sich für die meisten Völker der Sowjetunion die Industrialisierung und Urbanisierung des Landes aus, die die Grenzen der nationalen Verwaltungseinheiten durchbrechen und manchmal zu massenhaften Bevölkerungswanderungen führen. So stellen die Grossrussen heute in Kasachstan schon die grösste Volksgruppe (43,2\%), während ihr prozentueller Anteil an der Bevölkerung der übrigen Sowjetrepubliken zwischen 30,2\% (Kirgisistan) und 3,2\% (Armenien), durchschnittlich aber um 15\% liegt. Die aus wirtschaftlichen Gründen erfolgten Umsiedlungen führen meistens dazu, dass ein Nichtrusse in fremder Umgebung (etwa ein 'Moldavier' in Usbekistan) gezwungen ist, die russische Lingua franca zu benutzen und dadurch russifizierend mitwirkt. Wer eine höhere Funktion besonders im öffentlichen Dienst und in der Partei erstrebt, muss die russische Sprache perfekt beherrschen, so dass gerade die geistige Führungsschicht der nichtrussischen Völker, die Intelligenz, schon aus existenziellen Gründen gezwungen ist, Russisch als zweite Muttersprache zu pflegen, was natürlich auf die Dauer besonders bei den stammverwandten Slawenvölkern über die Zweisprachigkeit zur faktischen Ent-

24. Beachtenswert, aber nicht ganz zuverlässig E. J. Rozek, The Problems of National Minorities in the Soviet Union. Case Studies on Human Rights and Fundamental Freedoms, IV (Den Haag, 1976) $461 \mathrm{ff}$.

25. R.I. Rubin, ed., The Unredeemed Anti-Semitism in the Soviet Union (Chicago, 1968). Vgl. damit S.L. Burg, 'The Calculus of Soviet Antisemitism,' in Azrael, Soviet Nationality, 189 ff., und L. Kochan, ed., The Jews in Soviet Russia since 1917 (London, New York, Toronto, 1970). 
fremdung von der ursprünglichen Nationalität und zur Assimilierung führen muss. Die Sowjetstatistik verzeichnet in zunehmendem Masse Fälle, bei denen Angehörige einer nichtrussischen Nationalitat Russisch als ihre Muttersprache angeben. ${ }^{26}$ Dass diese verhängnisvollen Tendenzen die zahlenmässigen Verhältnisse zwischen den einzelnen Sowjetvölkern bis jetzt noch kaum zugunsten des Grossrussentums verschoben haben, ist hauptsächlich auf zwei Ursachen zurückzuführen. Es steht dem Sowjetbürger nicht frei, die Nationalität, die in seinem Personalausweiss angegeben ist, zu ändern. Eine solche Beschränkung schlägt sich natürlich in der Statistik konservativ nieder, während hinter der Fassade der Nationalitatsstatistik die schleichende Russifizierung fortschreitet. Die andere Ursache ist biologischer Art. Die Grossrussen haben, wie die meisten europäischen Sowjetvölker, heute eine niedrige Geburtenrate, ihre Volkszahl wächst jährlich mit etwa 0,6\%; inzwischen nimmt die asiatische Bevölkerung der Sowjetunion noch immer mit 3\% pro Jahr zu. Diese Zustände führen zu einem paradoxen Ergebnis. Einerseits sind von den gegenwärtigen 263 Millionen Sowjetbürgern schon beinahe zwei Drittel als Sprachrussen oder als zweisprachig zu betrachten, andererseits geht der Anteil der Grossrussen an der Gesamtbevölkerung ständig zurück und wird anno 1979 nur noch knapp die Hälfte betragen. Welche Folgen diese Entwicklung auf die Dauer haben wird, ist schwer vorherzusagen; meines Erachtens wird die sprachliche Assimilation fremdrussischer Elemente jedenfalls die sozialen Verhältnisse mit neuen Spannungen belasten und zwar in dem Sinne, dass bei den Grossrussen die Abwehrhaltung gegen die drohende 'Vergelbung' (ozeltenie) weiter zunehmen wird, während ihrerseits die zwar sprachlich assimilierten, aber sozial nicht als ebenbürtig akzeptierten Asiaten ihre Diskriminierung nicht langer passiv hinnehmen werden. Der Umstand, dass in der Dissidentenbewegung die nationale Frage eine immer grössere Rolle spielt, und auch die Unruhen, die sich in den letzten Jahren in der Ukraine, Litauen und Georgien ereignet haben, sind klare Warnzeichen einer wachsenden Unzufriedenheit der Nichtrussen in ihrer tatsächlichen Lage. ${ }^{27}$

26. Übrigens wird der offizielle Sowjetpatriotismus in zunehmendem Masse auch von den Grossrussen abgelehnt. S. Enders Wimbush, 'The Great Russians and the Soviet State,' in Azrael, Soviet Nationality, $349 \mathrm{ff}$.

27. E.G. Lewis, Multilingualism in the Soviet Union (Den Haag, 1972); ferner J. Azrael, 'Emergent Nationality Problems in the USSR,' in Azrael, Soviet Nationality, $363 \mathrm{ff}$. 
Union der Sozfafistischen Sowjetrepubliken Staatliche und ethnlsche Gliederung

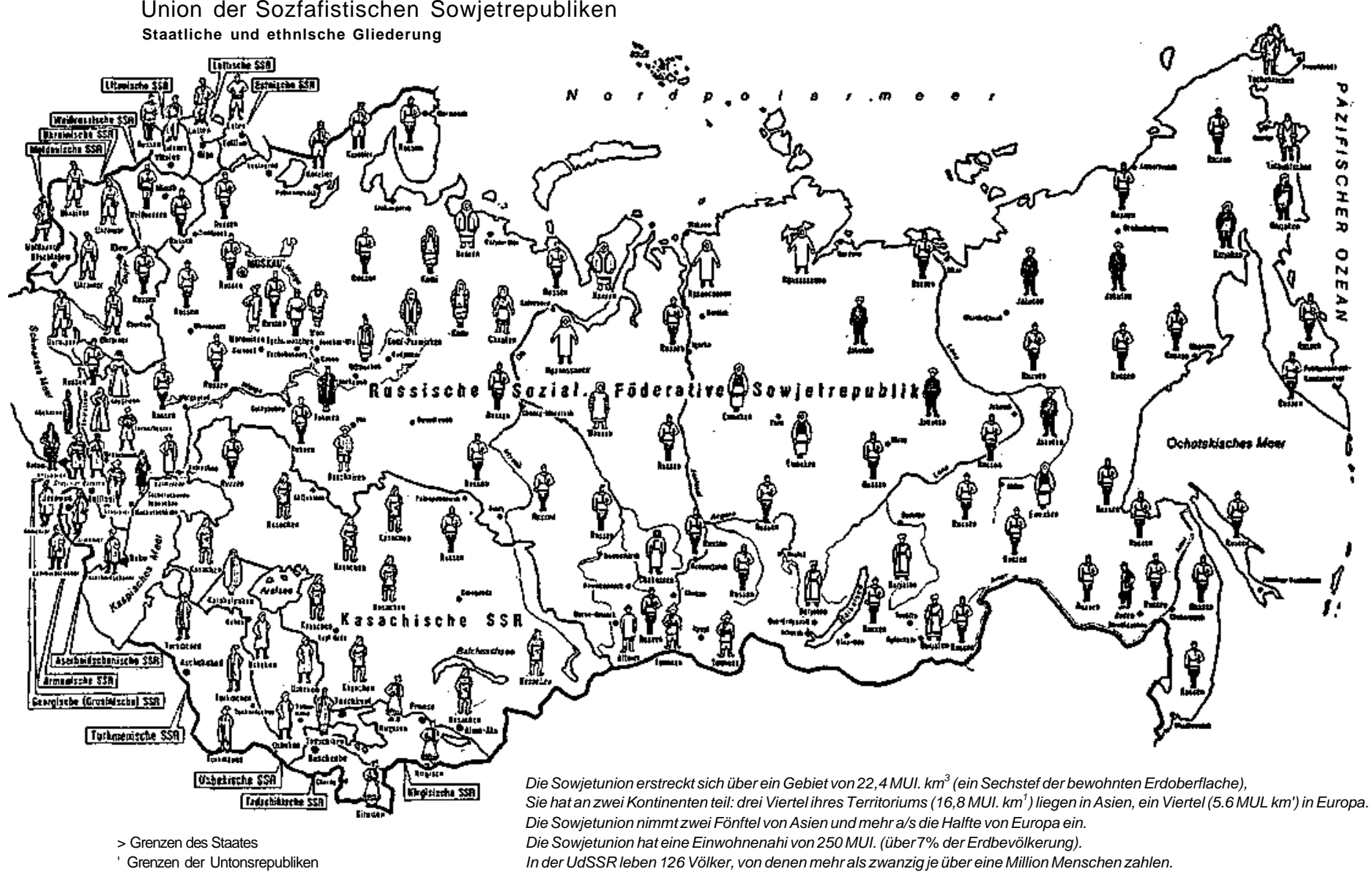

